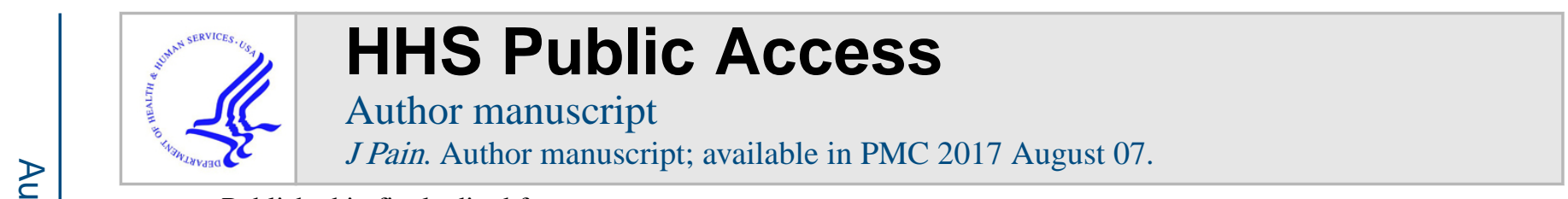

Published in final edited form as:

J Pain. 2013 April ; 14(4): 334-335. doi:10.1016/j.jpain.2013.01.007.

\title{
Reply to Commentary
}

Michael E. Robinson, PhD, Department of Clinical and Health Psychology, University of Florida, Gainesville, Florida

Roland Staud, MD, and

Department of Medicine, University of Florida, Gainesville, Florida

Donald D. Price, PhD

Department of Oral and Maxillofacial Surgery, University of Florida, Gainesville, Florida

The commentary by Sean Mackey ${ }^{3}$ makes several interesting points, many of which show strong agreement with the points raised by all of the other articles. Within the early part of his article he agrees that brain neuroimaging will not replace self-reporting of pain nor will it do so in the future and that central neuroimaging findings alone do not define pain as a disease. He then raises concerns that, based on our arguments forcefully presented to readers ${ }^{6}$ they "may lose sight of the real value of central neuroimaging-a way to augment self-report of pain and a potential, objective [italics ours] biomarker of pain and pain treatment." 3 There are several points we wish to make about Mackey's concerns that we think help clarify our agreements and disagreements.

Our commentary acknowledged what we consider to be real values of pain imaging, that of extending knowledge of mechanisms of pain, analgesia, and hyperalgesia. In fact, we have used this approach to a considerable extent in our own research. We further acknowledged that correlations between pain ratings and neuroimaging results "provide some external validation of pain ratings." However, a main point of our commentary is that the large proportion of validation of self-report measures remains within the domains of psychophysics (including quantitative sensory testing) and the rest of psychology. Sullivan et $\mathrm{al}^{7}$ make a similar and complementary point: "But brain imaging adds neuroanatomical and neurophysiological information, not validity, to pain reports." On this point we disagree slightly with Sullivan et $\mathrm{al}^{7}$ because we state that brain imaging has at least some role in externally validating self-report methods. However, we consider brain imaging to be a very important part of the science of pain, but most critically for understanding its mechanisms. We share Mackey's wish for readers not to lose sight of the value of central neuroimaging.

It is the idea that brain imaging can serve as a surrogate and objective marker of pain with which we respectfully disagree, and we think Mackey's discussion of this issue reverses his early claims of agreement with the other authors. For example, he states "However, no measure has provided a sufficiently high relationship with pain to be used as a valid surrogate for self-reports. Neuroimaging might be just such a surrogate" [italics ours]. ${ }^{3}$ If, as Mackey agrees earlier in his commentary, neuroimaging will not replace self-report, then how can it serve as a surrogate? The dictionary meaning of surrogate is replacement or substitute (thefreedictionary.com). A related point can be raised about neuroimages as "objective biomarkers." Do these biomarkers "mark" the pain itself or just the neural causes 
and correlates of pain? Measures of brain activity may certainly help understand mechanisms of pain, but could they replace self-report measures, as explicitly stated or implied elsewhere? ${ }^{1,4,9}$ The latter is at the heart of the ontology of pain (ie, pain is a type of experience) and, as pointed out in our commentary and elsewhere, causes and correlates do not establish ontological identity. 5,8

Mackey reports on new multivariate techniques (multivariate pattern analyses, and machine vector learning) as potential approaches to "mind reading." These are important developments in understanding brain function related to pain processing. However, consistent with our original contention, these multivariate techniques, like all previous techniques, require a "ground truth" to determine the accuracy of the classification derived from the mathematical models. The machine vector learning approach requires a "training sample" to develop the original algorithm. Of course, the "ground truth," or training data set, is the self-report of pain from the individual experiencing it. Though resulting high correct classification rates are touted as validation of these approaches, this must not be their purpose since self-report quite adequately identifies who has pain and where it is experienced.

We think there is a critically important difference between using neuroimaging to characterize neural representations and mechanisms of pain as opposed to using this method to provide substitutes, surrogates, or biomarkers of the actual experience of pain. And we don't think we are setting up a straw man. The 3 sets of authors we quoted in our original commentary explicitly claimed or directly implied that neural technologies will or should replace self-report of pain ${ }^{1,4,9}$ and some media interpretations of pain researchers emphasize this possibility. It is clear that all commentators agree that neuroimaging has an important place in the study of pain mechanisms. ${ }^{2,3,6,7,9}$ Where we think we continue to differ with others, ${ }^{1,4,9}$ particularly with Dr. Mackey, is whether neuroimaging could or should be used as a surrogate (ie, substitute) for self-report.

\section{References}

1. Apkarian AV, Hashmi JA, Baliki MN. Pain and the brain: specificity and plasticity of the brain in clinical chronic pain. Pain. 2011; 152(Suppl 3):S49-S64. [PubMed: 21146929]

2. Davis K. Is chronic pain a disease? Evaluating pain and nociception through self report and neuroimaging. J Pain. 2013; 14:332-333. [PubMed: 23548486]

3. Mackey S. Central neuroimaging of pain. J Pain. 2013; 14:328-331. [PubMed: 23548485]

4. NIH. Biomarkers for chronic pain using functional brain connectivity. 2011. commonfundnihgov

5. Price, DD., Barrell, JJ. Merging the two perspectives. MIT Press; 2012. Inner Experiences and Neuroscience.

6. Robinson ME, Staud R, Price DD. Pain measurement and brain activity: Will neuroimages replace pain ratings? J Pain. 2013; 14:323-327. [PubMed: 23548484]

7. Sullivan MD, Cahana A, Derbyshire S, Loeser JD. What does it mean to call chronic pain a brain disease? J Pain. 2013; 14:317-322. [PubMed: 23548483]

8. Velmans, M. Understanding Consciousness. Philadelphia, PA: Routledge/London; 2009.

9. Wartolowska K, Hough MG, Jenkinson M, Andersson J, Wordsworth BP, Tracey I. Structural brain changes in rheumatoid arthritis. Arthritis Rheum. 2012; 64:371-379. [PubMed: 21905009] 